\title{
Identifying Asymptomatic Patients at High-Risk for Stroke
}

\section{Kamran Gaba $^{1,2}$, Richard Bulbulia ${ }^{1,2}$ *}

${ }^{1}$ Medical Research Council Population Health Research Unit, Nuffield Department of Population Health, University of Oxford, UK

${ }^{2}$ Clinical Trial Service Unit and Epidemiological Studies Unit, Nuffield Department of Population Health, University of Oxford, UK

*Corresponding author: Richard Bulbulia, Medical Research Council Population Health Research Unit, Nuffield Department of Population Health, The University of Oxford, Richard Doll Building, Old Road Campus, Oxford, OX3 7LF, United Kingdom. E-mail: richard.bulbulia@ndph.ox.ac.uk

Notes: The Nuffield Department of Population Health (University of Oxford) and the Medical Research Council funded this research. There are no declared conflicts of interest. 


\begin{abstract}
Background: Carotid endarterectomy and carotid artery stenting, in addition to good medical therapy, halve long-term stroke risk in asymptomatic patients with carotid artery stenosis. Since the absolute benefits following successful intervention are moderate, identification of asymptomatic patients at high-risk of future stroke could maximise the effectiveness of carotid interventions.
\end{abstract}

Aim: To summarise the evidence for high-risk features associated with increased long-term stroke risk in asymptomatic patients.

Results: There is a paucity of reliable data describing the effect of clinical features, imaging findings and plaque characteristics on increased long-term stroke risk. Clinical and imaging features such as contralateral symptoms, silent brain infarcts/embolic signals, progression of stenosis and impaired cerebrovascular reactivity may be associated with increased future risk of stroke. Plaque characteristics such as echolucency, large plaque size ( $\geq 80 \mathrm{~mm})$, intra-plaque haemorrhage, lipid-rich necrotic core and thinned/ruptured fibrous cap may also increase future risk of stroke. Whilst these form the basis for European guidelines targeting carotid intervention in asymptomatic patients with tight stenosis, conclusive evidence of their utility is lacking.

Conclusions: Results from ongoing large, multi-centre randomised clinical trials comparing carotid endarterectomy and carotid artery stenting with good medical therapy may be consistent with earlier trials, showing a halving of the long-term risk of stroke following successful carotid revascularisation. However, they may well lack sufficient statistical power to identify higher-risk subgroups in whom the absolute gains of treatment are significantly higher. Large contemporary cohort studies are needed to provide further clarity regarding high-risk features associated with increased long-term stroke risk in asymptomatic patients with carotid artery stenosis. 
Key words: Carotid Endarterectomy; Carotid Artery Stenting; Asymptomatic; Carotid Artery Stenosis; Stroke 


\section{Introduction}

Stroke is a major cause of premature mortality and morbidity globally. The Global Burden of Disease study reported that, in 2010, cerebrovascular disease was the third most common cause of lost disability-adjusted life years (DALYs) globally (an increase of almost 20\% since 1990). ${ }^{1}$ Stroke is the fourth largest cause of death and a substantial cause of disability in the United Kingdom. ${ }^{2}$ More than 100,000 British adults have a stroke each year and more than 12,000 die within 30 days. $^{2}$ In America, stroke is the third largest cause of death, with $20-25 \%$ of strokes caused by carotid artery stenosis. ${ }^{3}$ Even when asymptomatic, carotid artery stenosis increases the relative risk (RR) of stroke by more than $50 \%{ }^{4}$

Successful carotid endarterectomy (CEA) or carotid artery stenting (CAS) halves long-term stroke risk. However, uncertainty still remains regarding the optimal technique for long-term stroke prevention in asymptomatic patients due to the moderate absolute benefits of CEA and CAS. ${ }^{5}$ There are also difficulties quantifying the long-term stroke risk in asymptomatic patients on good medical therapy due to small studies with wide confidence intervals and unrealistic adherence to intensive medical therapy with stringent follow-up that is unlikely to be replicated in routine clinical practice.${ }^{6,7}$ Furthermore, the majority of patients included had only moderate stenosis severity ${ }^{6,7}$, many of whom would be considered unsuitable for surgical or endovascular intervention.

The biological mechanism accounting for the reduction in annual rates of ipsilateral stroke and the extent to which contemporary medical therapy has had an effect remains unclear, especially since the rates of cigarette smoking have also been declining over the last few decades (a strong risk factor for future stroke). However, despite these reductions, stroke remains a major cause of avoidable premature death and major morbidity. Importantly, strokes related to carotid artery stenosis are serious, with $50 \%$ being fatal or disabling. The 
identification of patients with asymptomatic carotid artery stenosis who have high-risk features for future stroke may help maximise the effectiveness of carotid intervention.

This review will:

1. Briefly summarise the evidence and recommendations for carotid intervention in asymptomatic patients;

2. Critically appraise the use of high-risk features to select asymptomatic patients for carotid intervention who are at an increased likelihood of future stroke; and

3. Highlight areas for future research.

\section{CAROTID INTERVENTION IN ASYMPTOMATIC PATIENTS}

\section{CEA in Asymptomatic Patients: the evidence}

Three large randomised clinical trials (RCTs) comparing CEA (with good medical therapy) versus good medical therapy alone have shown that successful CEA halves long-term stroke risk in asymptomatic patients..$^{8-11}$

Between 1983 - 1987, the Veterans Affairs (VA) Cooperative Study recruited 444 patients and reported an absolute risk reduction in ipsilateral neurological events of $12.6 \%$ after CEA compared to good medical therapy $(8.0 \%$ versus $20.6 \%$ respectively; $\mathrm{p}<0.01)$, in patients with a mean follow-up of almost 4 years. ${ }^{8}$

Subsequently, between 1987 - 1993, the Asymptomatic Carotid Atherosclerosis Trial (ACAS) study recruited 1,662 patients and reported that CEA reduced the 5-year risk of the combined endpoint of ipsilateral stroke and any peri-operative stroke or death by $53 \%$ compared to good medical therapy (5.1\% versus $11.0 \%$ respectively; $p=0.004) .{ }^{9}$ 
Finally, between 1993 - 2003, the first Asymptomatic Carotid Surgery Trial (ACST-1) recruited 3,120 patients and reported a reduction in RR of stroke by approximately $50 \%$ following successful CEA. When including peri-operative risks, at 5 years, the rate of any stroke or peri-operative death was $6.9 \%$ following CEA and $10.9 \%$ on good medical therapy $(\mathrm{p}=0.0001) .{ }^{10,11}$ This was maintained out to 10 years $(\mathrm{p}=0.009) .{ }^{10,11}$ Procedural risks seen in old RCTs may not generalise to current clinical practice but the proportional reductions seen in stroke rate following successful CEA probably do generalise to the 2010's and beyond.

The main long-term findings of these RCTs are summarised in Table I, which show consistently that the risk of stroke is halved following successful CEA.

\section{CAS in Asymptomatic Patients: the evidence}

RCTs suggest that successful CEA and CAS may be equivalent in reducing long-term stroke risk in asymptomatic patients. ${ }^{12-16}$

The first Carotid Revascularisation Endarterectomy versus Stenting Trial (CREST-1) was a large multicentre RCT, with each surgeon and interventionalist required to meet a minimum standard for experience and performance. ${ }^{12}$ Embolic protection devices (EPDs) were used in $96.1 \%$ of patients. ${ }^{12}$ In asymptomatic patients $(n=1,181)$, the 4 -year rate of stroke or death (including the peri-procedural period) was 4.5\% (24 events) after CAS and 2.7\% (13 events) following CEA $(p=0.07) .{ }^{12}$ By 5 years, the post-procedural rate of stroke in asymptomatic patients was $2.5 \%$ after $\mathrm{CAS}$ and $2.7 \%$ following $\mathrm{CEA}^{13}$ and post-procedural ipsilateral stroke rates were similar out to 10 years, regardless of the modality of revascularisation used. $^{13}$ However, CREST-1 originally included symptomatic patients only, with 
asymptomatic patients included later. Hence, this trial was underpowered to reliably compare the effectiveness of CEA versus CAS in asymptomatic patients with carotid artery stenosis.

The Asymptomatic Carotid Trial-1 (ACT-1) trial recruited 1,453 patients and randomised them in a 3:1 ratio in favour of CAS compared to CEA. At 1 year, 3.8\% of patients receiving CAS and 3.4\% of patients undergoing CEA had died or experienced a stroke or myocardial infarction (MI). ${ }^{14}$ By 5 years, the ipsilateral stroke rate (excluding peri-procedural strokes) was $2.2 \%$ after CAS and $2.7 \%$ following CEA $(p=0.51)^{14}$, again suggesting equivalence between CEA and CAS for long-term stroke prevention.

The Stenting and Angioplasty with Protection in Patients at High Risk for Endarterectomy (SAPPHIRE) trial compared CAS with CEA in 334 patients selected for being at high operative risk (75.5\% of patients undergoing CEA had coronary artery disease). ${ }^{15}$ In 237 asymptomatic patients, the incidence of death, stroke or MI at 1 year was $9.9 \%$ in CAS patients and $21.5 \%$ in the CEA group and was statistically significant $(p=0.02) .{ }^{15}$ This is likely to be explained by the SAPPHIRE population having greater comorbidities, causing a significantly higher rate of procedural MI in the CEA compared to CAS group $(p=0.03) .{ }^{15}$ At 3 years, stroke rates were comparable between asymptomatic patients undergoing CEA or CAS (9.2\% and $10.3 \%$ respectively) and the combined endpoint of death, stroke or MI was also similar (29.2\% versus $21.4 \%$ respectively). ${ }^{16}$ SAPPHIRE's results should be interpreted with caution as patients were selected for being high-risk for CEA. As a result, long-term outcomes are dominated by a higher rate of procedural events than would be expected in both clinical practice at the time of the trial and by modern standards. Therefore, this RCT adds little useful information to help guide current management of asymptomatic patients.

The main long-term findings of the above RCTs are summarised in Table II. 
The evidence base directly comparing CEA and CAS in asymptomatic patients will be further clarified by the second Asymptomatic Carotid Surgery Trial (ACST-2) - the largest RCT ever conducted comparing CAS with CEA in asymptomatic patients with severe carotid artery stenosis on contemporary medical therapy (due to complete recruitment by early 2020). ${ }^{17}$ Reporting in 2021, initial results from ACST-2, describing early procedural risks and 4-year benefits of CEA versus CAS, will double the available evidence, providing much needed clarity and statistical power regarding procedural risks and long-term durability of CEA compared to CAS in patients taking contemporary medical therapy.

\section{European Guidelines for Carotid Intervention in Asymptomatic Patients}

When deciding whether a patient with asymptomatic carotid artery stenosis is suitable for intervention, it is important to balance the life-long benefits of a successful procedure against the 30-day procedural risks (including death, stroke and MI). Most RCTs have shown that the long-term risk of stroke is approximately halved by intervention. The procedural risks of CEA and CAS are reducing over time, but the background long-term risk of stroke in asymptomatic patients is also reducing due to improved medical therapy and lifestyle modification (e.g. smoking cessation). Therefore, careful patient selection for carotid intervention is necessary to maximise absolute benefits. This highlights the importance of identifying high-risk features that place asymptomatic patients at increased long-term risk of ipsilateral stroke.

Recent authoritative guidelines from the European Society for Vascular Surgery (ESVS), published in 2018, and European Society of Cardiology (ESC), published in 2017, recommend that patients with an asymptomatic carotid artery stenosis of $60-99 \%$ who are at 
'average surgical risk' should be considered for CEA or CAS (but CAS only if 'high surgical risk') if one or more characteristics are present that may be associated with an increased risk of late ipsilateral stroke. ${ }^{18,19}$ Previous history of contralateral Transient Ischaemic Attack (TIA) or stroke is a clinical feature that has been identified as increasing long-term risk of stroke on good medical therapy. ${ }^{18,19}$ Imaging criteria associated with an increased risk of stroke on good medical therapy include: silent infarction on cross-sectional brain imaging; stenosis progression; large plaque area; plaque echolucency; intra-plaque haemorrhage on Magnetic Resonance Imaging (MRI); impaired cerebral vasoreactivity; and/or spontaneous embolisation on Transcranial Doppler (TCD) Ultrasound. ${ }^{18,19}$ To derive net benefit, periprocedural risks should be low (less than 3\%) and the patient should have a life expectancy exceeding 5 years. ${ }^{18,19}$ The threshold in guidelines for acceptable procedural complication rates following CEA and CAS have decreased from $6 \%$ to $3 \% .{ }^{19,}{ }^{20}$ However, a threshold of $3 \%$ for procedural complications in asymptomatic patients may still be too high. A recent meta-analysis reported that the combined stroke/death rate following CEA in asymptomatic patients had halved since $2005(\mathrm{p}<0.001)$ but, in contrast, CAS outcomes remained similar $(\mathrm{p}=0.628) .{ }^{21}$ However, with greater experience and improvements in procedural developments (e.g. micro-mesh covered stents, trans-carotid arterial revascularisation (TCAR)), CAS procedural complications may well start to fall.

Features that predispose asymptomatic patients to an increased risk of stroke are summarised in Table III. These associations will be discussed in detail below, with critical appraisal of the evidence, consideration of biological plausibility and, perhaps most importantly, assessment of their clinical utility in selecting patients at high-risk of a carotid-related stroke who may derive particular benefit from intervention. 


\section{HIGH-RISK FEATURES FOR STROKE IN ASYMPTOMATIC PATIENTS}

\section{Prior Cerebral Infacrtion (Clinical or Image Detected) and Increased Stroke Risk}

Silent brain infarcts (SBIs) or previous stroke or TIA appear to be associated with increased long-term risk of stroke in asymptomatic patients. In a substudy of ACST- $1^{22}$, patients with SBIs or previous stroke or TIA $(n=1,331)$ compared to those without $(n=1,002)$ had significantly increased stroke risk at 5 years $(10.0 \%$ versus $7.0 \% ; p=0.01)$ and also at 10 years $(18.6 \%$ versus $12.8 \%$; Hazard Ratio (HR) $1.5(1.2-2.0) ; p=0.002){ }^{22}$ This is shown in Figure 1. Prior cerebral infarction was also associated with increased risk of fatal stroke within 10 years $(4.6 \%$ versus $2.9 \%$; $=0.009){ }^{22}$ This is biologically plausible as SBIs may reduce physiological reserve of neural tissue, predisposing it to higher future stroke risk with potentially fatal consequences. Also, a previously unstable plaque may be more likely to become unstable again in the future. These findings are statistically robust and were reported in the context of good triple medical therapy. They are therefore applicable to current medical practice and clinically useful.

The Asymptomatic Carotid Stenosis and Risk of Stroke (ACSRS) study was an international, multicentre cohort study that followed 1,115 patients with asymptomatic carotid artery stenosis of $50-99 \%$ (measured by the European Carotid Surgery Trial (ECST) method) for at least 5 years. $^{23}$ Patients with contralateral symptoms were included if they had been asymptomatic for 6 months prior to recruitment. A substudy of the ACSRS study were consistent with the ACST-1 substudy findings ${ }^{22}$ that SBIs were associated with increased long-term stroke risk in asymptomatic patients. In a subgroup of 821 patients with $60-99 \%$ stenosis, the cumulative stroke rate at 8 years was 19\% without SBIs but $37 \%$ with SBIs $(p=0.032) .{ }^{24}$ The corresponding stroke rate was $8 \%$ without but $29 \%$ with SBIs $(p=0.002) .{ }^{24}$ In patients with less than $60 \%$ stenosis, SBIs had no effect on stroke rates. ${ }^{24}$ In patients with 
$60-79 \%$ stenosis, the cumulative TIA and stroke rate was significantly higher in patients with SBIs than those without (35\% versus $10 \%$ respectively; $p=0.005) .{ }^{24}$ These findings suggest that SBIs increase future stroke risk in asymptomatic patients with moderate or severe stenosis. The substudy perhaps failed to detect an association between SBIs and future stroke risk in patients with mild stenosis (less than 60\%), possibly due to this subgroup being underpowered. It is also unclear how advancements in medical therapy over the last 10 years since the study was published may have affected this association.

The ACSRS study also reported that a history of contralateral TIA was independently associated with ipsilateral ischaemic events in asymptomatic patients (RR 3.0 (1.9-4.7); $\mathrm{p}=0.0001) .{ }^{23}$ This finding has been supported by older studies. ${ }^{25-28}$ Interestingly, the incidence of ipsilateral ischaemic events had a positive association with the degree of stenosis (ECST criteria). ${ }^{23}$ Age, gender, hypertension, cardiac symptoms, atrial fibrillation, diabetes, smoking, pack-years, cholesterol levels, body mass index or contralateral stenosis were not associated with increased risk of future stroke. ${ }^{23}$ The authors suggested that contralateral symptoms may be a surrogate for increased risk on the ipsilateral side, explaining the association. However, the ACSRS findings must be interpreted in the context of a small sample size, historical data and inclusion of patients at low future stroke risk. There were also only 108 ipsilateral events (18 amaurosis fugax, 44 TIAs and 46 ischaemic strokes) in the ACSRS study $^{23}$ raising the possibility that the association may be due to chance or confounding. 


\section{The Effect of TCD Embolic Signals on Increased Future Stroke Risk}

Frequent embolic signals on TCD appear to be associated with increased long-term stroke risk in asymptomatic patients. This was demonstrated by a meta-analysis that included 1,144 patients from the Asymptomatic Carotid Emboli Study (ACES) and older studies. ${ }^{29-34}$ ACES was a prospective observational study that recruited 482 patients with asymptomatic carotid artery stenosis of $70 \%$ or greater from 26 international centres. ${ }^{29}$ TCD recordings were obtained at baseline along with carotid duplex ultrasound and Computerised Tomography or MRI brain imaging. The meta-analysis reported that the presence of embolic signals at baseline was significantly associated with increased risk of ipsilateral stroke (HR 6.6 (2.915.4); $\mathrm{p}<0.0001$ ) and a composite of ipsilateral stroke and TIA (HR 7.6 (2.3-24.7); $\mathrm{p}=0.0008) .{ }^{29}$ However, the confidence intervals are wide in the latter outcome, probably reflecting heterogeneity in the analysis $(\mathrm{p}=0.002) .{ }^{29}$ Taken together, this suggests that embolic signals on TCD could be used to identify asymptomatic patients at increased risk of stroke who may derive maximum benefit from carotid intervention. This is biologically plausible as a positive association would be expected between the number of emboli and increased risk of future stroke. However, as TCD is not widely used in clinical practice, this limits the applicability of these findings.

The ACES study itself reported that the presence of embolic signals on TCD at baseline was significantly associated with an elevated risk of the combined endpoint of ipsilateral stroke and TIA within 2 years (HR $2.5(1.2-5.4) ; \mathrm{p}=0.015) .{ }^{29}$ The risk of stroke or TIA was similar whether the patient was taking anti-platelets at baseline or not. The risk of ipsilateral stroke alone was elevated more than 5-fold in patients with embolic signals at baseline (HR 5.6 (1.619.3); $\mathrm{p}=0.007) .{ }^{29}$ Again, baseline anti-platelet therapy had minimal effect. Therefore, TCD appears to be particularly good at detecting increased risk of future ipsilateral stroke. 
However, due to the small number of events $(n=10)$ and the resulting wide confidence interval $^{29}$, this raises the possibility that the association may be due to chance or confounding.

Other studies have also demonstrated an increased long-term stroke risk in asymptomatic patients with micro-emboli. Spence et al. ${ }^{30}$ reported an almost 18 -fold significantly increased risk of stroke in patients with micro-emboli compared to those without micro-emboli at 1 year $(p<0.0001) .{ }^{30}$ However, due to the small total number of events ( 8 TIAs and 8 strokes at 1 year in 319 patients, 1 TIA and 2 strokes at 2 years in 210 patients), this association should be interpreted with caution. A previous study by Spence et al. ${ }^{35}$ also demonstrated that the presence of micro-emboli significantly increased the risk of stroke at 1 and 2 years by more than 7-fold $(\mathrm{p}=0.02)$ and 10 -fold $(\mathrm{p}=0.001)$ respectively, with the use of good medical therapy associated with a significant reduction in stroke rate at 2 years $(\mathrm{p}=0.01)$. However, again, this study is limited by the small numbers of patients with micro-emboli $(n=37)$, the small number of events ( 8 strokes at 1 year in 468 patients and 10 strokes at 2 years in 468 patients) and the low proportion of patients on appropriate medical therapy (only $33.8 \%$ of patients recruited after 2003 were taking statins). ${ }^{35}$

\section{The Effect of Stenosis Progression on Future Stroke Risk}

Whilst there is no robust evidence exists to suggest that the severity of carotid artery stenosis predicts stroke risk in asymptomatic patients (unlike in symptomatic patients), stenosis progression has been identified as a factor that may be associated with an increased long-term stroke risk in asymptomatic patients. A further analysis of ACSRS in 1,121 patients with a mean follow-up of 4 years described that regression occurred in $3.8 \%$, progression in $19.8 \%$

and no change in $76.4 \%$ of carotid plaques. ${ }^{36}$ Increasing age, male gender, coronary artery 
disease, elevated serum creatinine and larger plaque area were all significantly associated with progression whereas use of lipid lowering agents was significantly associated with regression of carotid plaques. ${ }^{36}$ The overall 8 -year cumulative ipsilateral ischaemic stroke rate was $0 \%$ in patients with regression, $9 \%$ if the carotid plaque was unchanged in size and $16 \%$ in progression $(\mathrm{p}=0.05) .{ }^{36}$ The presence of plaque progression approximately doubled both the annual stroke rate and cumulative stroke rate at all degrees of stenosis between 5099\% (ECST method) and 70-99\% (North American Symptomatic Carotid Endarterectomy Trial method). ${ }^{36}$ This may account for the significantly reduced cerebral or retinal ischaemic event-rate survival $(\mathrm{p}<0.001)$ and stroke-free survival $(\mathrm{p}=0.05)$ in patients with carotid plaque progression compared with stable or regressing plaques. ${ }^{36}$ Statin use and their effect on plaque regression is well documented ${ }^{37}$ and findings from this study suggest that monitoring plaque changes may provide a means to assess the effectiveness of medical therapy in the future. This is biologically plausible as it would be expected that effective medical therapy would stabilise or reduce plaque size. Smaller plaque size may subsequently reduce future long-term stroke risk. However, as no robust evidence exists for this association, this will require validation in a larger population of patients (to reduce the possibility that the association is due to chance or confounding) before the findings of this study can be applied to routine clinical practice, limiting their utility.

\section{The Effect of Impaired Cerebrovascular Reactivity on Increased Future Stroke Risk}

Impaired cerebrovascular reactivity has been shown to increase future stroke risk. 'Cerebrovascular reactivity' is defined as a change in cerebral blood flow in response to a vasodilatory or vasoconstrictive stimulus. In 106 asymptomatic patients as part of a substudy of $\mathrm{ACES}^{38}$, TCD readings of the middle cerebral artery were measured to predict future 
stroke risk and a meta-analysis of similar studies was also performed to improve the power of their findings. ${ }^{38}$ The meta-analysis with 3 further studies ${ }^{39-41}$ showed that impaired cerebrovascular reactivity was associated with an increased risk of ipsilateral stroke or TIA (Odds Ratio (OR) 4.8 (1.9-12.1); $\mathrm{p}=0.001)$, ipsilateral stroke (OR 6.1 (1.3-29.5); $\mathrm{p}=0.02)$, any stroke (OR 4.7 (1.7-12.9); $\mathrm{p}=0.003)$ and any stroke or TIA (OR $1.6(1.9-10.9) ; \mathrm{p}=0.0006) .{ }^{38}$ Secondary analysis revealed that impaired cerebrovascular reactivity was associated with presence of embolic signals ${ }^{38}$, which may explain the strong association. However, these findings have uncertain clinical utility due to the limited use of TCD. Interestingly, the original ACES substudy showed no differences in TIA $(p=0.52)$ or stroke/TIA $(p=0.16)$ rates between patients with or without impaired cerebrovascular reactivity. ${ }^{38}$ This was likely due to the small size of the study and the small number of ipsilateral events (2 TIAs and 0 strokes in 106 patients) causing the results to be underpowered, despite ACES being the largest prospective study examining this association. The small sample size of both the original study and subsequent meta-analysis also raises the possibility that the association may be due to chance or confounding, limiting the applicability of findings.

\section{Ultrasonographic Features of Carotid Plaques and Increased Future Stroke Risk}

Various ultrasonographic features have been reported to be associated with increased longterm stroke risk in asymptomatic patients. The ACSRS group reported significantly reduced cerebral or retinal ischaemic event-free survival if plaques had more than 1 discrete white area $(\mathrm{p}<0.0001)$, a grayscale median less than $15(\mathrm{p}<0.0001)$ or size greater than or equal to

$80 \mathrm{~mm}(\mathrm{p}<0.0001)$ in 1,121 patients. $^{42}$ This was similar to findings from other studies. ${ }^{43,44}$ They also confirmed their previous findings that increased stenosis severity (ECST method) and history of contralateral TIA or stroke ( $p=0.002$ and $p<0.0001$ respectively) were both 
associated with reduced cerebral or retinal ischaemic event-free survival. ${ }^{42}$ This study was unique as it examined multiple different ultrasonic features of the plaque to determine future stroke risk. However, medical therapy was sub-optimal by modern standards throughout the study and $11.5 \%$ of their cohort of patients underwent CEA. This reduces the reliability of the reported results, requiring replication in larger studies (to reduce the possibility of the association being due to chance or confounding) before the findings can be applied clinically.

Juxtaluminal black areas (JBAs) are hypoechoic areas on ultrasound that indicate soft constituents of the plaque (e.g. lipid, haemorrhage and/or thrombus) adjacent to the lumen. They may represent unstable areas of the plaque that are at higher risk of rupture, increasing the risk of stroke. JBAs have been reported to increase long-term stroke risk in asymptomatic patients by the ACSRS authors in 1,121 patients. ${ }^{45}$ This was the first study to test this hypothesis, generated by reports of their proximity to the lumen in unstable, symptomatic plaques and that larger JBAs were associated with plaques becoming symptomatic. ${ }^{46,47}$ Increasing JBA size was independently associated with significantly increased 5-year cerebral or retinal ischaemic event rate, with average annual rates being $0.6 \%, 4.2 \%, 7.2 \%$ and $8.6 \%$ with JBA size $<4 \mathrm{~mm}^{2}, 4-8 \mathrm{~mm}^{2}, 8-10 \mathrm{~mm}^{2}$ and $>10 \mathrm{~mm}^{2}$ respectively $(\mathrm{p}<0.001) .{ }^{45}$ There was a similarly significantly elevated risk of stroke with each incremental increase in JBA size $(p<0.001) .{ }^{45}$ A cut off at $8 \mathrm{~mm}^{2}$ was able to identify patients at significantly increased risk of fatal stroke, both overall (OR 18.7 (4.1-85.9); $\mathrm{p}<0.001)$ and at 8 years $(\mathrm{HR}$ 19.6 (4.3-89.6); $\mathrm{p}<0.001) .{ }^{45}$ Despite the confidence intervals being wide suggesting a lack of power, the authors postulated that JBAs $>8 \mathrm{~mm}^{2}$ may produce a large ulcer, leading to a large embolus that would cause a large brain infarct and, ultimately, a stroke with significant consequences. However, measurement of JBAs is complex and error due to inter- and intraobserver variability can be introduced by calcification, vessel tortuosity and position. ${ }^{45}$ This reduces the reliability and validity of findings, limiting their clinical applicability. 
Plaque echolucency has also been associated with an increased risk of stroke in asymptomatic patients. This is biologically plausible as increased echolucency may be a marker for increased risk of ulceration of the plaque, leading to a stroke. A meta-analysis of 7,557 patients with asymptomatic carotid artery stenosis of $0-99 \%$ and a mean follow-up of 3 years showed a significant association between plaque echolucency and risk of future ipsilateral stroke $(\operatorname{RR} 2.3(1.6-3.4) ; \mathrm{p}<0.001) .{ }^{48}$ No heterogeneity $(\mathrm{p}=0.15)$ or publication bias $(\mathrm{p}=0.29)$ was reported. ${ }^{48}$ In a subgroup of patients with $50-99 \%$ stenosis $(n=2,095)$, the risk of future ipsilateral stroke was similarly elevated in those with echolucent plaques (RR 2.6 (1.5-4.6); $\mathrm{p}=0.001) .{ }^{48}$ However, there was a lack of consistency in determining echolucency in this study, requiring standardisation of methods. The restriction to only medically managed patients may have also limited the applicability of findings to patients undergoing CEA or CAS, who may be at higher future stroke risk. Whilst these findings may suggest an association between plaque echolucency and future stroke risk, the results need replication in a larger sample to reduce the possibility that the association is due to chance.

An observational, post-hoc analysis of 814 patients in the medical arm of the ACST-1 trial (i.e. restricted to those who did not receive CEA) also demonstrated that plaque echolucency was associated with increased risk of future stroke in asymptomatic patients. ${ }^{49}$ Patients with echolucent plaques $(n=403)$ had a significantly increased risk of ipsilateral stroke compared to those without $(n=411)$ at 5 years $(8.0 \%$ versus $3.1 \%$ respectively; $p=0.009) .{ }^{49}$ After adjustment for other risk factors, plaque echolucency remained significantly and independently associated with an increased risk of ipsilateral stroke (HR 2.5 (1.2-5.3); $\mathrm{p}=0.014) .{ }^{49}$ However, the overall risk of ipsilateral stroke at 10 years was similar whether the plaque was echolucent or not. ${ }^{49}$ Due to the small numbers of events captured in the 5-year analysis, the positive association needs to be reproduced in a larger population (with more events) to exclude the possibility that this may be a chance finding or due to an unidentified 
confounding factor. Increased statin use over time in ACST-1 may also explain why the association was not sustained at 10 years due to stabilisation of the carotid plaque. Alternatively, as higher-risk patients may be more likely to have had a stroke within the first 5 years, asymptomatic patients at 10 years may represent a self-selected lower-risk cohort (with more stable plaques), explaining why plaque echolucency had no effect beyond 5 years.

\section{MRI Features of High-Risk Carotid Plaques and Increased Future Stroke Risk}

Various MRI features have been associated with increased long-term stroke risk in asymptomatic patients. A meta-analysis in 678 patients with intra-plaque haemorrhage reported the risk of ipsilateral stroke and TIA was significantly elevated (HR 4.6 (2.9-7.2); $\mathrm{p}<0.01) .{ }^{50}$ In 403 patients with lipid-rich necrotic core, the risk of stroke and TIA was significantly increased (HR 3.0 (1.5-5.9); $\mathrm{p}=0.002) .{ }^{50}$ In 363 patients with thinned/ruptured fibrous cap, the risk of stroke and TIA was significantly raised (HR 5.9 (2.7-13.3); $<<0.01){ }^{50}$ These results were across all severities of carotid artery stenosis, showing that MRI plaque characteristics may be an independent risk factor for future stroke risk in asymptomatic patients. However, variability in MRI techniques for plaque imaging ${ }^{50}$ requires further validation to confirm that imaging findings are reproducible and reliable, limiting the clinical utility of the study's results. MRI studies carried out as part of the second Carotid Revascularisation and Medical Management for Asymptomatic Carotid Stenosis Trial $(\mathrm{CREST}-2)^{51,52}$ and the second European Carotid Surgery Trial (ECST-2) ${ }^{53}$ may provide further clarification.

The quality of the evidence presented and clinical utility of the features discussed above are summarised in Table IV. 


\section{FUTURE DIRECTIONS}

\section{Good Medical Therapy in Asymptomatic Patients}

Patients with asymptomatic carotid artery stenosis should all be managed with good medical therapy (i.e. intensive lipid-lowering therapy (usually high-dose statin), anti-platelet medication and a blood pressure modifying agent). ${ }^{54}$ Only a minority will undergo carotid intervention. However, medical therapy has improved over time and will continue to evolve. In the early years of recruitment in the ACST-1 trial, less than $10 \%$ of patients were taking lipid lowering therapy at baseline. ${ }^{11}$ This increased to $80 \%$ by the end of follow-up. ${ }^{11}$ As it has been demonstrated that a 1 mmol. $\mathrm{L}^{-1}$ reduction in low density lipoprotein cholesterol (LDL-C) reduces the 5-year risk of stroke by approximately $25 \%{ }^{55-57}$, increasing statin use may partly explain why background stroke risk has been falling over the last few decades. Simvastatin has been shown to be particularly effective in reducing carotid-related stroke, halving the rate of CEA or CAS in symptomatic patients in the Heart Protection Study. ${ }^{55}$

The ESVS (2018) and ESC (2017) guidelines recommend that a healthy diet, smoking cessation and physical activity should all be instituted in patients with asymptomatic carotid artery stenosis. ${ }^{18,19}$ Use of low-dose aspirin was also recommended. Whilst aspirin has not been shown to reduce the risk of stroke in a primary prevention setting, the rationale for recommending aspirin for patients with asymptomatic carotid artery stenosis is due to its cardio-protective effects, but this should be balanced against an increased risk of haemorrhage.

Lipid-lowering therapy (most commonly statins) has also been recommended for long-term prevention of cardiovascular disease in asymptomatic patients with a tight carotid artery stenosis. ${ }^{18,58}$ Attempts should be made to achieve targets of either a 50\% reduction in LDL-C 
or serum levels $<1.8 \mathrm{mmol} . \mathrm{L}^{-1}\left(70 \mathrm{mg} . \mathrm{dL}^{-1}\right)^{20,59,60}$, reductions which are readily achievable with modern statin therapy (i.e. $40-80 \mathrm{mg}$ atorvastatin or $20-40 \mathrm{mg}$ rosuvastatin). The Cholesterol Treatment Trialists' Collaborators ${ }^{59}$ meta-analysis of 14 RCTs of statins (including 90,056 individuals) reported a significant $21 \%$ reduction in all major vascular events $(\mathrm{p}<0.0001) .{ }^{59}$ There was a similar significant reduction in fatal or non-fatal stroke (RR $0.8(0.8-0.9) ; \mathrm{p}<0.0001) .{ }^{59}$ Even more intensive lipid lowering may be possible in the future with the use of proprotein convertase subtilisin/kexin type 9 (PCSK-9) inhibitors (e.g. alirocumab or evolocumab). These monoclonal antibodies, administered via subcutaneous injection every 2-4 weeks, inhibit the enzyme PCSK-9. This inhibition causes more LDL receptors to be presented to the surface of hepatocytes, increasing LDL-C metabolism. They have been shown to reduce serum LDL-C levels by $60 \%$ in patients already taking statins in the ODYSSEY and FOURIER trials. ${ }^{61-63}$ In the recent FOURIER trial comparing evolocumab to placebo (with background statin therapy in both groups), the composite rate of cardiovascular death, MI or stroke was significantly reduced by $20 \%$ (HR 0.8 (0.7-0.9); $\mathrm{p}<0.001$ ) in 27,564 patients with prior cardiovascular disease. ${ }^{63}$ Ischaemic stroke rate was also significantly reduced (HR $0.8(0.6-0.9) ; \mathrm{p}=0.01) .{ }^{63}$ However, the efficacy and safety of PCSK-9 inhibitors beyond 4 years follow-up is unknown. Their effects in patients with asymptomatic carotid artery stenosis are also uncertain but it seems likely that these agents will further reduce the long-term stroke risk when they begin to be prescribed more widely.

Observational data linking elevated systolic blood pressure with increased risk of stroke is strong. However, the Systolic Blood Pressure Intervention Trial (SPRINT) failed to demonstrate a significant reduction in strokes in asymptomatic patients with a systolic blood pressure $<120 \mathrm{mmHg}$ compared to those $<140 \mathrm{mmHg}(\mathrm{HR} 0.9(0.6-1.3) ; \mathrm{p}=0.50) .{ }^{64}$ Intensive blood pressure treatment was significantly associated with hypotension and impaired renal function (both $\mathrm{p}<0.001$ ), compared to standard treatment. ${ }^{64}$ This raises the concern that 
overly intensive blood pressure lowering may have deleterious effects in patients with tight carotid artery stenosis who may depend on their systemic vascular resistance for brain perfusion to overcome a tight stenosis that may be reducing flow and causing cerebral hypoperfusion. However, treatment of hypertension is associated with reduction and regression of carotid plaques in asymptomatic patients. ${ }^{65} \mathrm{~A}$ meta-analysis reported a reduction in stroke risk proportional to lowering of systolic blood pressure and a Chinese RCT demonstrated reduced stroke risk with use of enalapril. ${ }^{66,67}$ The pharmacological agent used is less important than achieving the target of reducing blood pressure $<140 / 90 \mathrm{mmHg}$ in non-diabetic patients. ${ }^{68}$ In diabetic patients, intensive blood pressure control has been shown to reduce the RR of stroke by $44 \%$ compared to patients with a higher blood pressure. ${ }^{69}$ Therefore, the joint European Society for Hypertension/ESC guidelines (2013) recommend that blood pressure in diabetic patients with asymptomatic carotid artery stenosis should be $<140 / 85 \mathrm{mmHg}{ }^{70}$

In diabetic patients with asymptomatic carotid artery stenosis, tight glycaemic control is recommended as these patients are at increased risk of stroke. ${ }^{71}$ It is also important that medical therapy is appropriately prescribed. In a non-randomised study of patients with type II diabetes, the use of lipid-lowering therapy, anti-platelet agents and anti-hypertensive medications was associated with a $60 \%$ reduction in cardiovascular events and deaths. ${ }^{72}$

As part of good medical therapy in CREST-2, participants receive intensive anti-hypertensive and lipid-lowering therapies, with novel medications not covered by medical insurance provided to patients for free if they are not achieving their therapeutic targets (e.g. PCSK-9 inhibitors are provided to patients already on maximum dose of statins who are above their LDL-C target). ${ }^{51}$ Goal-directed therapy targets ${ }^{52}$ include systolic blood pressure $<140 \mathrm{mmHg}$ and serum LDL-C $<70 \mathrm{mg} \cdot \mathrm{dL}^{-1}$. Participants are also enrolled in a lifestyle modification programme (INTERVENT) that targets weight loss, smoking cessation, increased exercise, 
reduced alcohol intake and diabetes management. ${ }^{51}$ A lifestyle coach regularly telephones them (every 2 weeks for the first 3 months, then 3-monthly until 1 year, then 6-monthly until 4 years) to ensure adherence to INTERVENT. ${ }^{51}$ This will help better define the effect of contemporary medical therapy on stroke risk in asymptomatic patients (though it may not be feasible to apply this resource-intensive programme to routine clinical practice).

\section{Advancements in Anti-Thrombotic Therapies}

Novel medical therapies may further reduce long-term stroke risk in asymptomatic patients.

Addition of low-dose rivaroxaban to aspirin has shown promising results. The Cardiovascular

Outcomes for People using Anticoagulation Strategies (COMPASS) trial $^{73}$ investigated the effect of adding low-dose rivaroxaban to aspirin in 7,470 patients with peripheral arterial disease. The composite endpoint of cardiovascular death, MI or stroke was significantly reduced (HR $0.7(0.6-0.9) ; \mathrm{p}=0.0047)$ with addition of low-dose rivaroxaban as were major adverse limb events, including amputation (HR $0.5(0.4-0.8) ; \mathrm{p}=0.0037){ }^{73}$ A subgroup analysis of 1,919 patients with previous carotid artery revascularisation or asymptomatic carotid artery stenosis of at least $50 \%$ showed that the overall major adverse cardiovascular event rate was reduced (HR $0.6(0.4-1.1) ; \mathrm{p}=0.07)$ with addition of low-dose rivaroxaban. ${ }^{73}$ This was not at the expense of increased risk of haemorrhage. ${ }^{73}$ Whilst this subgroup analysis was inadequately powered to provide meaningful conclusions, larger RCTs may reveal a beneficial effect of adding low-dose rivaroxaban to aspirin in long-term stroke prevention.

In patients presenting with acute coronary syndromes, the Study of Platelet Inhibition and Patient Outcomes (PLATO) reported no significant difference in stroke risk $(1.5 \%$ versus $1.3 \%$; HR $1.2(0.9-1.5) ; \mathrm{p}=0.22)$ in patients prescribed ticagrelor $(\mathrm{n}=9,333)$ versus 
clopidogrel $(n=9,291)$ respectively at 12 months. ${ }^{74}$ However, there was a $21 \%$ reduction in death from vascular causes $(p=0.001)$ and $16 \%$ reduced recurrent MI rate $(p=0.005)$ which must be balanced against a 10-fold increased risk of fatal intracranial haemorrhage $(p=0.02) .{ }^{74}$ Further studies should be conducted to clarify whether the addition of ticagrelor to patients with asymptomatic carotid artery stenosis reduces their long-term stroke risk.

Ticagrelor has also been shown to significantly reduce recurrent ischaemic stroke risk in patients presenting with a non-severe ischaemic stroke or TIA in the Acute Stroke or Transient Ischaemic Attack Treated with Aspirin or Ticagrelor and Patient Outcomes (SOCRATES) trial. $^{75}$ Recurrent 90-day ischaemic stroke risk (5.8\% versus $6.7 \%$; HR 0.9 $(0.8-1.0) ; \mathrm{p}=0.046)$ and all stroke risk $(5.9 \%$ versus $6.8 \%$; HR $0.9(0.8-1.0) ; \mathrm{p}=0.03)$ was significantly reduced following treatment with ticagrelor $(n=6,589)$ than aspirin $(n=6,610)$ respectively. ${ }^{75}$ Benefit was more pronounced in patients with minor ischaemic strokes (HR $0.8(0.7-1.0))$ and there was no increased risk of haemorrhage. ${ }^{75}$ Whilst these analyses were in symptomatic patients, future studies may report primary prevention benefits of prescribing ticagrelor to patients with asymptomatic carotid artery stenosis.

Ticagrelor has not shown benefit over clopidogrel in the Examining Use of Ticagrelor in Peripheral Artery Disease (EUCLID) trial. ${ }^{76}$ In 13,885 patients with symptomatic peripheral artery disease, randomised to ticagrelor $(n=6,930)$ or clopidogrel $(6,955)$, there was no significant difference in the composite outcome of cardiovascular death, MI or ischaemic stroke at 3 years $(10.8 \%$ versus $10.6 \%$ respectively; HR $1.0(0.9-1.1) ; p=0.65) .{ }^{76}$ However, there was a significant reduction in ischaemic stroke associated with ticagrelor use compared to clopidogrel $(1.9 \%$ versus $2.4 \%$; HR $0.8(0.6-1.0)$; $\mathrm{p}=0.03)$, with no increase in the risk of intracranial $(p=0.82)$ or fatal haemorrhage $(p=0.10) .{ }^{76}$ This suggests that ticagrelor may be beneficial in reducing the long-term risk of stroke. 


\section{Procedural Considerations}

A significant risk factor for procedural complications following CEA or CAS is an unsafe surgeon or interventionalist. Over time, familiarity and experience has increased with both surgical techniques and catheter-based procedures so carotid interventions are now safer than at any time previously. Continually improving medical therapy, patient selection with preprocedural optimisation and technological advancement will likely further improve future CEA and CAS outcomes. In particular, improvements in stent design (e.g. micro-mesh covered stents), delivery systems (e.g. TCAR) and EPDs may reduce CAS complications.

When deciding whether to perform a carotid intervention in an asymptomatic patient, it is important to consider life expectancy. To compensate for the initial procedural hazards of stroke and/or death, it takes approximately 4 years for the benefits of intervention to exceed the protective effects of medical therapy. Life expectancy must exceed this period to justify intervention in this group of patients whose absolute long-term risks of stroke are low. This provides the rationale for the ESVS (2018) and ESC (2017) guidelines recommending a life expectancy of 5 years as a pre-requisite for performing carotid interventions in asymptomatic

patients. ${ }^{18,19}$ People are living longer and those who may reasonably expect to live for 10 or more good quality years may enjoy even greater absolute gains from carotid intervention.

\section{$\underline{\text { Future Perspectives }}$}

Ongoing RCTs such as CREST-2 and ECST-2 will compare CAS (with use of EPDs) and CEA with good medical therapy in asymptomatic patients with severe carotid artery stenosis whilst the ACTRIS trial will compare CEA plus good medical therapy versus good medical therapy alone. ${ }^{77}$ However, analyses of the medical therapy groups within these RCTs may 
lack sufficient statistical power to identify higher-risk subgroups in whom the absolute gains of treatment are significantly higher. Instead, large contemporary cohort studies are needed to provide further clarity regarding high-risk features associated with increased long-term stroke risk in asymptomatic patients with carotid artery stenosis.

The United Kingdom Carotid Cohort Study (UKCCS) is one such study. It is a prospective observational study that aims to follow $5,000-10,000$ asymptomatic patients for over 10 years. This will be the largest ever cohort of asymptomatic patients (with a broad range of carotid artery stenoses) studied over a long time, taking contemporary medical therapy. Findings from UKCCS may therefore provide sufficient power to allow detection of high-risk features associated with increased long-term stroke risk in asymptomatic patients.

\section{Conclusions}

Whilst CEA and CAS have been shown to reduce long-term stroke risk in asymptomatic patients with carotid artery stenosis significantly, absolute benefits are moderate. It is therefore important to identify high-risk subgroups of asymptomatic patients who may be at increased risk of future strokes to maximise the absolute benefits of carotid intervention. Clinical and imaging features such as contralateral symptoms, SBIs, stenosis progression, embolic signals on TCD and impaired cerebrovascular reactivity may be associated with increased future stroke risk. Plaque characteristics such as echolucency, size, intra-plaque haemorrhage, lipid-rich necrotic core and thinned/ruptured fibrous cap may also increase future stroke risk. Whilst these have formed the basis for European guidelines targeting carotid intervention in asymptomatic patients with tight stenosis, life expectancy exceeding 5 years and at least one of the above features, conclusive evidence of their actual clinical utility 
is still lacking. Large contemporary cohort studies such as UKCCS should provide further clarity regarding high-risk features associated with increased long-term stroke risk in asymptomatic patients with carotid artery stenosis.

\section{Source(s) of Funding and Conflict(s) of Interest/Disclosures}

The Nuffield Department of Population Health (University of Oxford) and the Medical Research Council funded this research. There are no declared conflicts of interest. 


\section{References}

1. Murray CJL, Vos T, Lozano R, Naghavi M, Flaxman AD, Michaud C, et al. Disability-adjusted life years (DALYs) for 291 diseases and injuries in 21 regions, 1990-2010: a systematic analysis for the Global Burden of Disease Study 2010. Lancet 2012; 380 (9859): 2197-2223.

2. Stroke Association. State of the nation: Stroke statistics, 2018. Available at: https://www.stroke.org.uk/system/files/sotn_2018.pdf (accessed 30 August 2018).

3. Roger VL, Go AS, Lloyd-Jones DM, Adams RJ, Berry JD, Brown TM, et al. Heart disease and stroke statistics - 2011 update: a report from the American Heart Association. Circulation 2011; 123 (4): e18-e209.

4. Aichner FT, Topakian R, Alberts MJ, Bhatt DL, Haring HP, Hill MD, et al. REACH Registry Investigators. High cardiovascular event rates in patients with asymptomatic carotid stenosis: the REACH Registry. Eur J Neurol 2009; 16 (8): 902-908.

5. Gaba K, Ringleb PA, Halliday A. Asymptomatic Carotid Stenosis: Intervention or Best Medical Therapy? Curr Neurol Neurosci Rep 2018; 18 (11): 80.

6. Abbott AL. Medical (nonsurgical) intervention alone is now best for prevention of stroke associated with asymptomatic severe carotid stenosis: results of a systematic review and analysis. Stroke 2009; 40 (10): e573-583.

7. Marquardt L, Geraghty OC, Mehta Z, Rothwell PM. Low risk of ipsilateral stroke in patients with asymptomatic carotid stenosis on best medical treatment: a prospective, population-based study. Stroke 2010; 41 (1): e11-17.

8. Hobson RW, Weiss DG, Fields WS, Goldstone J, Moore WS, Towne JB, et al. Efficacy of carotid endarterectomy for asymptomatic carotid stenosis. N Engl J Med 1993; 328: 221-227.

9. ACAS Investigators. Endarterectomy for asymptomatic carotid artery stenosis. Executive Committee for the Asymptomatic Carotid Atherosclerosis Study. JAMA 1995; 273: 1421-1428. 
10. Halliday A, Mansfield A, Marro J, Peto C, Peto R, Potter J, et al. Prevention of disabling and fatal strokes by successful carotid endarterectomy in patients without recent neurological symptoms: randomised controlled trial. Lancet 2004; 363: 1491-1502.

11. Halliday A, Harrison M, Hayter E, Kong X, Mansfield A, Marro J, et al. 10-year stroke prevention after successful carotid endarterectomy for asymptomatic stenosis (ACST-1): a multicentre randomised trial. Lancet 2010; 376 (9746): 1074-1084.

12. Brott TG, Hobson II RW, Howard G, Roubin GS, Clark WM, Brooks W, et al. for the CREST Investigators. Stenting versus endarterectomy for treatment of carotid artery stenosis. $N$ Engl $J$ Med 2010; 363 (1): 11-23.

13. Brott TG, Howard G, Roubin GS, Meschia JF, Mackey A, Brooks W, et al. for the CREST Investigators. Long-term results of stenting versus endarterectomy for carotid artery stenosis. $N$ Engl J Med 2016; 374 (11): 1021-1031.

14. Rosenfield K, Matsumura JS, Chaturvedi S, Riles T, Ansel GM, Metzger C, et al. The ACT I Investigators. Randomised trial of stent versus surgery for asymptomatic carotid stenosis. $N$ Engl J Med 2016; 374: 1011-1020.

15. Yadav JS, Wholey MH, Kuntz RE, Fayad P, Katzen BT, Mishkel GJ, et al. for the Stenting and Angioplasty with Protection in Patients at High Risk for Endarterectomy Investigators. Protected carotid artery stenting versus endarterectomy in high-risk patients. N Engl J Med 2004; 351 (15): 1493-1501.

16. Gurm HS, Yadav JS, Fayad P, Katzen BT, Mishkel GJ, Bajwa TK, et al. for the SAPPHIRE Investigators. Long-term results of carotid stenting versus endarterectomy in high-risk patients. $N$ Engl J Med 2008; 358 (15): 1572-1579.

17. Halliday A, Bulbulia R, Gray W, Naughten A, den Hartog A, Delmestri A, et al. ACST-2 Collaborative Group. Status update and interim results from the asymptomatic carotid surgery trial-2 (ACST-2). Eur J Vasc Endovasc Surg 2013; 46 (5): 510-518. 
18. Naylor AR, Ricco JB, de Borst GJ, Debus S, de Haro J, Halliday A, et al. Editor's Choice Management of atherosclerotic Carotid and Vertebral Artery disease: 2017 clinical practice guidelines of the European Society for Vascular Surgery (ESVS). Eur J Vasc Endovasc Surg 2018; 55 (1): 3-81.

19. Aboyans V, Ricco J-B, Bartelink M-LEL, Björck M, Brodmann M, Cohnert T, et al. 2017 ESC guidelines on the diagnosis and treatment of peripheral arterial diseases, in collaboration with the European Society for Vascular Surgery (ESVS): Document covering atherosclerotic disease of extracranial carotid and vertebral, mesenteric, renal, upper and lower extremity arteries. Endorsed by: the European Stroke Organisation (ESO). The Task Force for the Diagnosis and Treatment of Peripheral Arterial Diseases of the European Society of Cardiology (ESC) and of the European Society for Vascular Surgery (ESVS). Eur Heart J 2018; 39 (9): 763-816.

20. Tendera M, Aboyans V, Bartelink M-L, Baumgartner I, Clément D, Collet J-P, et al. ESC guidelines on the diagnosis and treatment of peripheral artery diseases: document covering atherosclerotic disease of extracranial carotid and vertebral, mesenteric, renal, upper and lower extremity arteries: the Task Force on the Diagnosis and Treatment of Peripheral Artery Diseases of the European Society of Cardiology (ESC). Eur Heart J 2011; 32 (22): 2851-2906.

21. Lokuge K, de Waard DD, Halliday A, Gray A, Bulbulia R, Mihaylova B. Meta-analysis of the procedural risks of carotid endarterectomy and carotid artery stenting over time. Br J Surg 2018; 105 (1): 26-36.

22. Streifler JY, den Hartog AG, Pan S, Pan H, Bulbulia R, Thomas DJ, et al. Ten-year risk of stroke in patients with previous cerebral infarction and the impact of carotid surgery in the Asymptomatic Carotid Surgery Trial. Int J Stroke 2016; 11 (9): 1020-1027.

23. Nicolaides AN, Kakkos SK, Griffin MB, Sabetai MM, Dhanjil S, Tegos T, et al. Severity of Asymptomatic Carotid Stenosis and Risk of Ipsilateral Hemispheric Ischaemic Events: Results from the ACSRS Study. Eur J Vasc Endovasc Surg 2005; 30 (3): 275-284. 
24. Kakkos SK, Sabetai MM, Tegos T, Stevens J, Thomas DJ, Griffin MB, et al. Silent embolic infarcts on computed tomography brain scans and risk of ipsilateral hemispheric events in patients with asymptomatic internal carotid artery stenosis. J Vasc Surg 2009; 49 (4): 902-909.

25. Norris JW, Zhu CZ, Bornstein NM, Chambers BR. Vascular risks of asymptomatic carotid stenosis. Stroke 1991; 22 (12): 1485-1490.

26. Zhu CZ, Norris JW. A therapeutic window for carotid endarterectomy in patients with asymptomatic carotid stenosis. Can J Surg 1991; 34 (5): 437-440.

27. Mackey AE, Abrahamowicz M, Langlois Y, Battista R, Simard D, Bourque F, et al. Outcome of asymptomatic patients with carotid disease. Asymptomatic Cervical Bruit Study Group. Neurology 1997; 48 (4): 896-903.

28. Nadareishvili ZG, Rothwell PM, Beletsky V, Pagniello A, Norris JW. Long-term risk of stroke and other vascular events in patients with asymptomatic carotid artery stenosis. Arch Neurol 2002; 59 (7): 1162-1166.

29. Markus HS, King A, Shipley M, Topakian R, Cullinane M, Reihill S, et al. Asymptomatic embolisation for prediction of stroke in the Asymptomatic Carotid Emboli Study (ACES): a prospective observational study. Lancet Neurol 2010; 9 (7): 663-671.

30. Spence JD, Tamayo A, Lownie SP, Ng WP, Ferguson GG. Absence of microemboli on transcranial Doppler identifies low-risk patients with asymptomatic carotid stenosis. Stroke 2005; 36 (11): 2373-2378.

31. Abbott AL, Chambers BR, Stork JL, Levi CR, Bladin CF, Donnan GA. Embolic signals and prediction of ipsilateral stroke or transient ischaemic attack in asymptomatic carotid stenosis: a multicentre prospective cohort study. Stroke 2005; 36 (6): 1128-1133.

32. Molloy J, Markus HS. Asymptomatic embolisation predicts stroke and TIA risk in patients with carotid artery stenosis. Stroke 1999; 30 (7): 1440-1443. 
33. Orlandi G, Fanucchi S, Sartucci F, Murri L. Can microembolic signals identify unstable plaques affecting symptomatology in carotid stenosis? Stroke 2002; 33 (7): 1744-1746.

34. Siebler M, Nachtmann A, Sitzer M, Rose G, Kleinschmidt A, Rademacher J, et al. Cerebral microembolism and the risk of ischaemia in asymptomatic high-grade internal carotid artery stenosis. Stroke 1995; 26 (11): 2184-2186.

35. Spence JD, Coates V, Li H, Tamayo A, Muñoz C, Hackam DG, et al. Effects of intensive medical therapy on microemboli and cardiovascular risk in asymptomatic carotid stenosis. Arch Neurol 2010; 67 (2): 180-186.

36. Kakkos SK, Nicolaides AN, Charalambous I, Thomas DJ, Giannopoulos A, Naylor AR, et al. Predictors and clinical significance of progression or regression of asymptomatic carotid stenosis. J Vasc Surg 2014; 59 (4): 956-967.

37. Hegland O, Kurz MW, Munk PS, Larsen JP. The effect of statin therapy on the progression of carotid artery stenosis in relation to stenosis severity. Acta Neurol Scand 2010; 121 (1): 11-15.

38. King A, Serena J, Bornstein NM, Markus HS. Does Impaired Cerebrovascular Reactivity Predict Stroke Risk in Asymptomatic Carotid Stenosis? A Prospective Substudy of the Asymptomatic Carotid Emboli Study. Stroke 2011; 42 (6): 1550-1555.

39. Gur AY, Bova I, Bornstein NM. Is impaired cerebral vasomotor reactivity a predictive factor of stroke in asymptomatic patients? Stroke 1996; 27 (12): 2188-2190.

40. Markus HS, Cullinane M. Severely impaired cerebrovascular reactivity predicts stroke and TIA risk in patients with carotid artery stenosis and occlusion. Brain 2001; 124 (Pt 3): 457-467.

41. Silvestrini M, Vernieri F, Pasqualetti P, Matteis M, Passarelli F, Troisi E, et al. Impaired cerebral vasoreactivity and risk of stroke in patients with asymptomatic carotid artery stenosis. JAMA 2000; 283 (16): 2122-2127. 
42. Nicolaides AN, Kakkos SK, Kyriacou E, Griffin MB, Sabetai MM, Thomas DJ, et al. Asymptomatic internal carotid artery stenosis and cerebrovascular risk stratification. J Vasc Surg 2010; 52 (6): 1486-1496.

43. Mathiesen EB, Bønaa KH, Joakimsen O. Echolucent plaques are associated with high risk of ischaemic cerebrovascular events in carotid stenosis: The Tromsø Study. Circulation 2001; 103 (17): 2171-2175.

44. Hashimoto H, Tagaya M, Niki H, Etani H. Computer-assisted analysis of heterogeneity on Bmode imaging predicts instability of asymptomatic carotid plaque. Cerebrovasc Dis 2009; 28 (4): 357-364.

45. Kakkos SK, Griffin MB, Nicolaides AN, Kyriacou E, Sabetai MM, Tegos T, et al. The size of juxtaluminal hypoechoic area in ultrasound images of asymptomatic carotid plaques predicts the occurrence of stroke. J Vasc Surg 2013; 57 (3): 609-618.

46. Bassiouny HS, Sakaguchi Y, Mikucki SA, McKinsey JF, Piano G, Gewertz BL, et al. Juxtaluminal location of plaque necrosis and neoformation in symptomatic carotid stenosis. $J$ Vasc Surg 1997; 26 (4): 585-594.

47. Griffin MB, Kyriacou E, Pattichis C, Bond D, Kakkos SK, Sabetai MM, et al. Juxtaluminal hypoechoic area in ultrasonic images of carotid plaques and hemispheric symptoms. J Vasc Surg 2010; 52 (1): 69-76.

48. Gupta A, Kesavabhotla K, Baradaran H, Kamel H, Pandya A, Giambrone AE, et al. Plaque Echolucency and Stroke Risk in Asymptomatic Carotid Stenosis: A Systematic Review and MetaAnalysis. Stroke 2015; 46 (1): 91-97.

49. Huibers A, de Borst GJ, Bulbulia R, Pan H, Halliday A. ACST-1 collaborative group. Plaque Echolucency and the Risk of Ischaemic Stroke in Patients with Asymptomatic Carotid Stenosis Within the First Asymptomatic Carotid Surgery Trial (ACST-1). Eur J Vasc Endovasc Surg 2016; 51 (5): 616-621. 
50. Gupta A, Baradaran H, Schweitzer AD, Kamel H, Pandya A, Delgado D, et al. Carotid plaque MRI and stroke risk: a systematic review and meta-analysis. Stroke 2013; 44 (11): 3071-3077.

51. Mott M, Koroshetz W, Wright CB. CREST-2: Identifying the Best Method of Stroke Prevention for Carotid Artery Stenosis: National Institute of Neurological Disorders and Stroke Organisational Update. Stroke 2017; 48 (5): e130-e131.

52. Howard VJ, Meschia JF, Lal BK, Turan TN, Roubin GS, Brown RD Jr, et al. CREST-2 study investigators. Carotid revascularisation and medical management for asymptomatic carotid stenosis: Protocol of the CREST-2 clinical trials. Int J Stroke 12 (7): 770-778.

53. The $2^{\text {nd }}$ European Carotid Surgery Trial (ECST-2) Investigators. The $2^{\text {nd }}$ European Carotid Surgery Trial (ECST-2) Protocol, 2015. Available at: http://s489637516.websitehome.co.uk/ECST2/downloads/ECST-2\%20Protocol\%20v\%203_1.pdf

54. Paraskevas KI, Mikhailidis DP, Veith FJ, Spence JD. Definition of best medical treatment in asymptomatic and symptomatic carotid artery stenosis. Angiology 2016; 67 (5): 411-419.

55. Collins R, Armitage J, Parish S, Sleight P, Peto R. The Heart Protection Study Collaborative Group. Effects of cholesterol-lowering with simvastatin on stroke and other major vascular events in 20536 people with cerebrovascular disease or other high-risk conditions. Lancet 2004; 363 (9411): 757-767.

56. Heart Protection Study Collaborative Group. Randomized trial of the effects of cholesterollowering with simvastatin on peripheral vascular and other major vascular outcomes in 20,536 people with peripheral vascular disease and other high-risk conditions. J Vasc Surg 2007; 45 (4): 653-654.

57. Amarenco P, Bogousslavsky J, Callahan A $3^{\text {rd }}$, Goldstein LB, Hennerici M, Rudolph AE, et al. The Stroke Prevention by Aggressive Reduction in Cholesterol Levels (SPARCL) Investigators. High-dose atorvastatin after stroke or transient ischaemic attack. New Engl J Med 2006; 355 (6): 549-559. 
58. Taylor F, Huffman MD, Macedo AF, Moore THM, Burke M, Davey Smith G, et al. Statins for the primary prevention of cardiovascular disease. Cochrane Database Syst Rev 2013; (1): CD004816.

59. Baigent C, Keech A, Kearney PM, Blackwell L, Buck G, Pollicino C, et al. Cholesterol Treatment Trialists' (CTT) Collaborators. Efficacy and safety of cholesterol-lowering treatment: prospective meta-analysis of data from 90,056 participants in 14 randomised trials of statins. Lancet 2005; 366 (9493): 1267-1278.

60. Stone NJ, Robinson JG, Lichtenstein AH, Bairey Merz CN, Blum CB, Eckel RH, et al. American College of Cardiology/American Heart Association Task Force on Practice Guidelines. 2013 ACC/AHA guideline on the treatment of blood cholesterol to reduce atherosclerotic cardiovascular risk in adults: a report of the American College of Cardiology/American Heart Association Task Force on Practice Guidelines. J Am Coll Cardiol 2014; 63 (25 Pt B): 28892934.

61. Sabatine MS, Giugliano RP, Wiviott SD, Raal FJ, Blom DJ, Robinson J, et al. Open-Label Study of Long-Term Evaluation against LDL Cholesterol (OSLER) Investigators. Efficacy and safety of evolocumab in reducing lipids and cardiovascular events. N Engl J Med 2015; 372 (16): 15001509.

62. Robinson JG, Farnier M, Krempf M, Bergeron J, Luc G, Averna M, et al. ODYSSEY LONG TERM Investigators. Efficacy and safety of alirocumab in reducing lipids and cardiovascular events. N Engl J Med 2015; 372 (16): 1489-1499.

63. Sabatine MS, Giugliano RP, Keech AC, Honarpour N, Wiviott SD, Murphy SA, et al. FOURIER Steering Committee and Investigators. Evolocumab and Clinical Outcomes in Patients with Cardiovascular Disease. N Engl J Med 2017; 376 (18): 1713-1722. 
64. Wright JT Jr, Williamson JD, Whelton PK, Snyder JK, Sink KM, Rocco MV, et al. SPRINT Research Group. A Randomised Trial of Intensive versus Standard Blood-Pressure Control. $N$ Engl J Med 2015; 373 (22): 2103-2116.

65. Sutton-Tyrrell K, Wolfson SK Jr, Kuller LH. Blood pressure treatment slows the progression of carotid stenosis in patients with isolated systolic hypertension. Stroke 1994; 25 (1): 44-50.

66. Law MR, Morris JK, Wald NJ. Use of blood pressure lowering drugs in the prevention of cardiovascular disease: meta-analysis of 147 randomised trials in the context of expectations from prospective epidemiological studies. BMJ 2009; 338: b1665.

67. Huo Y, Li J, Qin X, Huang Y, Wang X, Gottesman RF, et al. CSPPT Investigators. Efficacy of folic acid therapy in primary prevention of stroke among adults with hypertension in China: the CSPPT randomized clinical trial. JAMA 2015; 313 (13): 1325-1335.

68. Neal B, MacMahon S, Chapman N. Blood Pressure Lowering Treatment Trialists' Collaboration. Effects of ACE inhibitors, calcium antagonists, and other blood-pressure-lowering drugs: results of prospectively designed overviews of randomised trials. Blood Pressure Lowering Treatment Trialists' Collaboration. Lancet 2000; 356 (9246): 1955-1964.

69. UK Prospective Diabetes Study Group. Tight blood pressure control and risk of macrovascular and microvascular complications in type 2 diabetes: UKPDS 38. BMJ 1998; 317 (7160): 703-713.

70. ESH/ESC Task Force for the Management of Arterial Hypertension. 2013 practice guidelines for the management of arterial hypertension of the European Society of Hypertension (ESH) and the European Society of Cardiology (ESC): ESH/ESC Task Force for the Management of Arterial Hypertension. J Hypertens 2013; 31 (10): 1925-1938.

71. Banerjee C, Moon YP, Paik MC, Rundek T, Mora-McLaughlin C, Vieira JR, et al. Duration of diabetes and risk of ischaemic stroke: the Northern Manhattan Study. Stroke 2012; 43 (5): 12121217. 
72. Gaede P, Lund-Andersen H, Parving HH, Pedersen O. Effect of a multifactorial intervention on mortality in type 2 diabetes. $N$ Engl J Med 2008; 358 (6): 580-591.

73. Anand SS, Bosch J, Eikelboom JW, Connolly SJ, Diaz R, Widimsky, et al. Rivaroxaban with or without aspirin in patients with stable peripheral or carotid artery disease: an international, randomised, double-blind, placebo-controlled trial. Lancet 2018; 391: 219-229.

74. Wallentin L, Becker RC, Budaj A, Cannon CP, Emanuelsson H, Held C, et al. Ticagrelor versus Clopidogrel in Patients with Acute Coronary Syndromes. N Engl J Med 2009; 361 (11): 10451057.

75. Johnston SC, Amarenco P, Albers GW, Denison H, Easton JD, Evans SR, et al. SOCRATES Steering Committee and Investigators. Ticagrelor versus Aspirin in Acute Stroke or Transient Ischaemic Attack. N Engl J Med 2016; 375 (1): 35-43.

76. Hiatt WR Fowkes FGR, Heizer G, Berger JS, Baumgartner I, Held P, et al. EUCLID Trial Steering Committee and Investigators. Ticagrelor versus Clopidogrel in Symptomatic Peripheral Artery Disease. N Engl J Med 2017; 376 (1): 32-40.

77. Gaba K, Halliday A. Epidemiology and indications for revascularization of carotid stenoses. In: Faggioli G, Halliday A, Pini R, Gallitto E, Stella A. Carotid Disease: Clinical and morphological insights. Turin: Edizioni Minerva Medica S.p.A.; 2017. 
Table I: Summary of the evidence for the role of CEA in long-term stroke prevention, compared to medical therapy, in asymptomatic patients

\begin{tabular}{|c|c|c|c|c|c|}
\hline $\begin{array}{c}\text { Clinical } \\
\text { Trial }\end{array}$ & $\begin{array}{c}\text { Years of } \\
\text { Recruitment }\end{array}$ & $\begin{array}{l}\text { Degree of } \\
\text { Stenosis }\end{array}$ & $\begin{array}{l}\text { Number of } \\
\text { Patients }\end{array}$ & $\begin{array}{c}\text { Follow-up } \\
\text { (years) }\end{array}$ & Findings \\
\hline VA & 1983-1987 & $\geq 50 \%$ & 444 & 4.0 & $\begin{array}{l}\text { Ipsilateral TIA, amaurosis fugax or stroke } \\
\text { CEA: } 8.0 \% \text {, Medical Therapy: } 20.6 \% \text {; } \mathbf{p}<\mathbf{0 . 0 0 1}\end{array}$ \\
\hline ACAS & $1987-1993$ & $\geq 60 \%$ & 1,662 & 2.7 & $\begin{array}{l}\text { Peri-procedural stroke or death, and post-operative ipsilateral stroke } \\
\text { CEA: } 5.1 \% \text {, Medical Therapy: } 11.0 \% ; \mathbf{p = 0 . 0 0 4}\end{array}$ \\
\hline ACST-1 & 1993-2003 & $\geq 60 \%$ & 3,120 & 10.0 & $\begin{array}{c}\text { Any stroke or peri-operative death } \\
\text { CEA: } 6.9 \% \text {, Medical Therapy: } 10.9 \% ; \mathbf{p}=\mathbf{0 . 0 0 0 1} \\
\text { Any stroke or peri-operative death } \\
\text { CEA: } 13.4 \% \text {, Medical Therapy: } 17.9 \% ; \mathbf{p}=\mathbf{0 . 0 0 9}\end{array}$ \\
\hline
\end{tabular}

Abbreviations - ACAS: Asymptomatic Carotid Atherosclerosis Study; ACST-1: Asymptomatic Carotid Surgery Trial 1; CEA: Carotid Endarterectomy; TIA: Transient Ischaemic Attack; VA: Veterans Affairs Co-operative Study 
Table II: Summary of the evidence for the role of CAS in long-term stroke prevention, compared to CEA, in asymptomatic patients

\begin{tabular}{|c|c|c|c|c|}
\hline $\begin{array}{c}\text { Clinical } \\
\text { Trial }\end{array}$ & $\begin{array}{c}\text { Years of } \\
\text { Recruitment }\end{array}$ & $\begin{array}{l}\text { Number of } \\
\text { Patients }\end{array}$ & $\begin{array}{c}\text { Follow-up } \\
\text { (years) }\end{array}$ & Findings \\
\hline CREST-1 ${ }^{*}$ & 2000-2008 & 1,181 & 10.0 & $\begin{array}{l}\text { Peri-procedural MI, stroke, death and post-procedural ipsilateral stroke } \\
\qquad \text { CEA: } 5.4 \% \text {, CAS: } 6.1 \% ; \mathbf{p = 0 . 9 5} \\
\text { Peri-procedural MI, stroke, death and post-procedural ipsilateral stroke } \\
\text { CEA: } 10.1 \% \text {, CAS: } 9.6 \%\end{array}$ \\
\hline ACT-1 & 2005-2013 & 1,453 & 5.0 & $\begin{array}{l}\text { Post-procedural ipsilateral stroke } \\
\text { CEA: } 2.7 \% \text {, CAS: } 2.2 \% ; \mathbf{p}=\mathbf{0 . 5 1}\end{array}$ \\
\hline SAPPHIRE * & 2000-2002 & 237 & 3.0 & $\begin{array}{l}\text { Peri-procedural MI, stroke, death and post-procedural ipsilateral stroke and death } \\
\qquad \text { CEA: } 29.2 \% \text {, CAS: } 21.4 \%\end{array}$ \\
\hline
\end{tabular}

Abbreviations - ACT-1: Asymptomatic Carotid Stenosis 1; CAS: Carotid Artery Stenting; CEA: Carotid Endarterectomy; CREST-1: Carotid Revascularisation Endarterectomy versus Stenting Trial; MI: Myocardial Infarction; SAPPHIRE: Stenting and Angioplasty with Protection in Patients at High Risk for Endarterectomy

* denotes subgroup analysis 
Table III: Risk factors for increased long-term stroke risk in asymptomatic patients, identified by ESVS (2018) and ESC (2017) guidelines ${ }^{18,19}$

\section{Characteristic}

Clinical

Cerebral Imaging

Ultrasound Imaging

Plaque Features on MRI

\section{Risk Factor}

Contralateral TIA/stroke

Silent infarctions on CT

Embolic signals on Transcranial Doppler

Stenosis progression

Impaired cerebrovascular reactivity

Large, echolucent plaques

Increased juxta-luminal black areas

Intra-plaque haemorrhage

Lipid-rich necrotic core

Abbreviations - CT: Computerised Tomography; ESC: European Society of Cardiology; ESVS: European Society for Vascular Surgery; MRI: Magnetic Resonance Imaging; TIA: Transient Ischaemic Attack 
Table IV: Strength of the evidence and clinical utility of the features associated with increased long-term stroke risk in asymptomatic patients

$\begin{array}{llll}\text { Feature } & \text { Evidence } & \text { Clinical Utility } \\ \text { Silent infarctions on CT } & \underline{\text { Moderate }} & & \text { Potentially useful } \\ \text { Large, echolucent plaques } & \underline{\text { Moderate }} & & \text { Potentially useful } \\ \text { Contralateral TIA/stroke } & \text { Weak } & \text { Potentially useful } \\ \text { Stenosis progression } & \text { Weak } & \text { Potentially useful } \\ \text { Embolic signals on Transcranial Doppler } & \text { Weak } & \text { Not useful } \\ \text { Impaired cerebrovascular reactivity } & \text { Weak } & \text { Not useful } \\ \text { Increased juxtaluminal black areas } & \text { Weak } & \text { Not useful } \\ \text { Intra-plaque haemorrhage } & & \text { Weak } & \text { Not useful } \\ \text { Lipid-rich necrotic core } & & \text { Weak } & \text { Not useful }\end{array}$




\section{Captions}

Table I: Summary of the evidence for the role of CEA in long-term stroke prevention, compared to medical therapy, in asymptomatic patients

Table II: Summary of the evidence for the role of CAS in long-term stroke prevention, compared to CEA, in asymptomatic patients

Table III: Risk factors for increased long-term stroke risk in asymptomatic patients, identified by ESVS (2018) and ESC (2017) guidelines ${ }^{18,19}$

Table IV: Strength of the evidence and clinical utility of the features associated with increased long-term stroke risk in asymptomatic patients

Figure 1: Differences in 5-year and 10-year risks of stroke, comparing patients with a history of ischaemic stroke or TIA or radiological evidence of an asymptomatic brain infarct (Group 1) versus patients without previous cerebral infarction (Group 2), in a substudy of the ACST-1 trial 